\title{
Pharmacokinetics of coadministration of levothyroxine sodium and alendronate sodium new effervescent formulation
}

\author{
H. G. Bone ${ }^{1}$ (D) - M. A. Walter ${ }^{2}$ - M. E. Hurley ${ }^{3}$ - S. Epstein ${ }^{4}$
}

Received: 30 November 2016 / Accepted: 23 January 2017 /Published online: 16 February 2017

(C) The Author(s) 2017. This article is published with open access at Springerlink.com

\begin{abstract}
Summary No clinically important pharmacokinetic interference of alendronate occurred between a new effervescent formulation of alendronate and levothyroxine when coadministered. The combination does not materially affect levothyroxine absorption. Introduction Concurrent treatment of osteoporosis with alendronate (Aln) and hypothyroidism with levothyroxine (LT4) may be problematic because both drugs are to be taken separately after fasting overnight. The primary objective was to assess pharmacokinetic interactions between a new effervescent formulation of Aln (Aln-NEF) and LT4.

Methods A randomized, open-label, 3-way crossover study was conducted in 30 healthy adults (15 women). Subjects were dosed 3 times, separated by 35 days, after overnight fasts, with Aln-NEF alone $(70 \mathrm{mg})$, LT4 alone $(600 \mu \mathrm{g})$, or Aln-NEF and LT4 concurrently. Samples were analyzed for plasma Aln and serum LT4. Pharmacokinetic drug-drug interaction was assessed using $90 \%$ confidence intervals (CIs) for the test/reference ratio of the geometric means for area under the concentration-time curve from time zero to last measureable time point $\left(\mathrm{AUC}_{0-t}\right)$ and maximum concentration
\end{abstract}

H. G. Bone

hgbone.md@att.net

1 Michigan Bone \& Mineral Clinic, 22201 Moross Road, Suite 260, Detroit, MI 48236, USA

2 Mission Pharmacal Company, 10999 Interstate Highway 10 West, Suite 1000, San Antonio, TX 78230, USA

3 Hurley Consulting Associates Ltd., 25 DeForest Avenue, Summit, NJ 07901, USA

4 Division of Endocrinology Diabetes and Bone Disease, Icahn School of Medicine at Mt. Sinai, Atran Building, 1428 Madison Avenue, 4th Floor, Box 1055, New York, NY 10029, USA
$\left(C_{\text {max }}\right)$. Results were compared to the default no-effect boundaries of 80 to $125 \%$ for the ratio Aln-NEF and LT4 concurrently/Aln-NEF alone and the ratio Aln-NEF and LT4 concurrently/LT4 alone.

Results Geometric mean ratios (Aln-NEF with LT4/Aln-NEF alone) were 0.927 (90\% CI 0.795-1.081) for $\mathrm{AUC}_{0-8}$ and 0.912 (90\% CI 0.773-1.077) for $C_{\max }$, demonstrating LT4 does not appreciably affect the pharmacokinetics of Aln. Geometric mean ratios (LT4 with Aln-NEF/LT4 alone) were 1.049 (90\% CI 0.983-1.119) for $\mathrm{AUC}_{0-48}$ and 1.075 (90\% CI 1.006-1.148) for $C_{\max }$, demonstrating LT4 is bioequivalent between the 2 treatments. Coadministration of Aln-NEF and LT4 was well tolerated.

Conclusions There was no clinically important pharmacokinetic interference between the Aln-NEF formulation and LT4. Aln-NEF does not materially affect LT4 absorption.

Keywords Alendronate - Bisphosphonate - Drug interaction · Levothyroxine $\cdot$ Pharmacokinetics

\section{Introduction}

Alendronate (Aln) is a widely used bisphosphonate for treatment of osteoporosis in postmenopausal women and for treatment to increase bone mass in men with osteoporosis [1,2]. A recently approved new effervescent formulation of Aln (AlnNEF) is given once weekly. As with all Aln formulations, AlnNEF must be taken after an overnight fast at least $30 \mathrm{~min}$ before the first food, beverage, or medication of the day because food decreases the absorption. The dosing instructions for Aln-NEF must be followed in order to assure adequate drug absorption and to minimize the risk of esophageal adverse reactions [3]. 
It may be challenging for a patient to take more than 1 prescribed medication in the fasted state. A search was performed to identify oral drugs taken in the fasted state [4]. The search identified six drugs that are to be taken exclusively under overnight fasting conditions: three bisphosphonates (Aln alone and in combination with cholecalciferol, ibandronate, and risedronate), levothyroxine (LT4), and linaclotide (a guanylate cyclase-C agonist used to treat chronic constipation). Many patients treated for osteoporosis may require thyroid hormone replacement. A study conducted in older adults ( 57 to 85 years of age) to estimate potential major drug-drug interactions of medications and supplements using a household interview documented the use of these medications [5]. Aln and LT4 were the 15th and 4th most commonly used drugs, respectively. It was estimated that $4.5 \%$ of older adults in the USA take Aln and 12.4\% take LT4 [5].

\section{Pharmacokinetics}

According to the approved product labeling, Aln has low oral bioavailability, and there is a well-documented decrease in its absorption when taken with food [3]. Clinical studies with Aln oral tablets (Fosamax ${ }^{\circledR}$ ) showed the mean oral bioavailability relative to intravenous administration of Aln in women was $0.64 \%$ (for doses ranging from 5 to $70 \mathrm{mg}$ ) and $0.59 \%$ (for a dose of $10 \mathrm{mg}$ ) in men when administered after an overnight fast and $2 \mathrm{~h}$ before breakfast [3]. Aln was effective when administered at least $30 \mathrm{~min}$ before breakfast in studies of the treatment and prevention of osteoporosis [3].

Aln administration with coffee or orange juice reduced bioavailability by approximately $60 \%$ [3].

Aln-NEF has also demonstrated a food interaction. A study evaluating the effect of food on absorption showed Aln bioavailability to be decreased by approximately $50 \%$ when AlnNEF was administered 15 min before a standardized breakfast to 119 healthy women compared to Aln-NEF administration followed by a 4-h fast [3].

LT4 is administered as a single daily dose, preferably one half to $1 \mathrm{~h}$ before breakfast and should be taken at least $4 \mathrm{~h}$ apart from drugs that are known to interfere with its absorption. LT4 is administered in the fasted state because certain foods alter its bioavailability. Gastrointestinal tract absorption of LT4 ranges from 40 to $80 \%$ and is increased by fasting and decreased by certain foods. LT4 has a narrow therapeutic index. Careful dosage titration is necessary in order to avoid the consequences of over- or under-treatment. Many drugs interact with LT4 necessitating adjustments in dosing to maintain an optimal therapeutic response; however, bisphosphonates including Aln are not listed as interacting drugs in the LT4 prescribing information [6].

\section{Evaluation of potential interaction}

Potential interaction between LT4 and Aln could be due to chemical reaction, interaction of the excipients in the formulations, effects on the metabolism of the drugs, effects on protein binding, effects on renal excretion, and effects on pharmacodynamics.

Neither LT4 nor Aln possesses reactive substituents and both are negatively charged; therefore, they would not be expected to interact chemically when ingested together. Interference from excipients in the formulations is unlikely. The LT4 tablets used in this study do not contain materials known to interfere with Aln absorption in sufficient quantity to be of concern, and the Aln-NEF formulation does not contain any of the materials known to decrease LT4 absorption [3, 6]. Because Aln is not metabolized, it is not expected to affect metabolizing enzymes and the metabolism of other drugs including LT4 [6]. Other potential clinically important pharmacokinetic interactions involving protein binding and renal excretion are also considered unlikely due to the small amounts of the two drugs dosed and/or absorbed [3, 6]. Any interference between Aln-NEF and LT4 would likely be related to interference in absorption.

\section{Study design considerations}

The primary objective was to evaluate the pharmacokinetic interaction between Aln-NEF and LT4. Evaluation of the safety and tolerability of coadministration of Aln-NEF and LT4 was a secondary objective. The design of the Aln and LT4 study is complicated because Aln is poorly absorbed and circulating levels are low. Furthermore, in order to raise LT4 levels above the endogenous background in normal subjects to allow measurement, several times a normal dose of LT4 must be given, and the washout period for LT4 is long (35 days). The dose administered in this study is a multiple of the highest dose of LT4 marketed in keeping with FDA guidance on LT4 tablets for in vivo bioavailability studies.

The use of a large dose $(600 \mu \mathrm{g})$ of Synthroid in this study is based on the Synthroid application for marketing approval to FDA (NDA 21-402). It was established that two 300- $\mu \mathrm{g}$ tablets were bioequivalent to a $600-\mu \mathrm{g}$ oral solution [7]. The Synthroid application also showed proportionality between 50-, 100-, and 300- $\mu$ g Synthroid tablets. The approved product label for Synthroid states that the excipients are the same for each strength [6]. In order to deliver $600 \mu \mathrm{g}$ of LT4 while simulating the clinical administration of only one or two tablets, the use of two 300- $\mu$ g tablets was chosen for this study to minimize the risk of any artifact that might be introduced by a large number of tablets.

The sample size was based on FDA guidelines [8] and not on statistical considerations. FDA's Division of Bone, Reproductive, and Urologic Products was consulted 
concerning the design of the study and the advice was followed.

\section{Materials and methods}

\section{Study participants}

This study was conducted in 15 men and 15 nonpregnant, nonlactating women, aged 18 to 45 years, who were healthy based on a complete physical examination and medical history, vital signs, electrocardiography (ECG), and clinical laboratory tests. Subjects were not enrolled if they had a history of thyroid disorders, esophageal disorders, and/or swallowing disorders; had participated in another clinical trial with an investigational drug within 30 days before inclusion in this trial; or had taken any prescription or non-prescription drugs within the 2 weeks preceding the study; if they had smoked, chewed tobacco, or used nicotine-containing products within the past 12 months; or had consumed alcohol within $24 \mathrm{~h}$ prior to screening. Subjects had to be able to sit or stand upright for $30 \mathrm{~min}$. A number of restrictions were imposed on the subjects, including abstinence from alcohol, caffeine-containing beverages, tobacco (or nicotine-containing products), and highly spiced foods for the duration of the study. On the day of administration, the subjects were not allowed to eat any food other than that provided.

All subjects gave written informed consent. The study was approved by Chesapeake Institutional Review Board; it was conducted in compliance with the good clinical practice and ethical standards for human experimentation and in accordance with applicable regulatory requirements. The study was conducted between December 2013 and March 2014 at Celerion, Neptune, NJ, and was sponsored by Mission Pharmacal Company.

\section{Study design}

This was a randomized, single-center, open-label, 3-way crossover study in healthy men and women. Subjects received 3 treatments in random sequence. There was a 35-day washout following the first 2 study periods. Discharge from the study occurred $48 \mathrm{~h}$ after the third study period.

Study drugs were administered to subjects after a $12-\mathrm{h}$ overnight fast. Subjects received either 1 dose of Aln-NEF $70 \mathrm{mg}$ effervescent tablet (Binosto®, Mission Pharmacal Company) dissolved in $4 \mathrm{oz}$ water, 1 dose of LT4 $600 \mu \mathrm{g}$ $(2 \times 300 \mu \mathrm{g}$ Synthroid $\AA$, AbbVie Inc.) tablets with $4 \mathrm{oz}$ water, or concomitantly 1 dose of LT4 $600 \mu \mathrm{g}(2 \times 300 \mu \mathrm{g})$ tablets with 1 dose of Aln-NEF $70 \mathrm{mg}$ dissolved in $4 \mathrm{oz}$ water, in a crossover design. According to the Aln-NEF label, the pharmacy waited at least $5 \mathrm{~min}$ after the effervescence stopped and stirred the solution for approximately $10 \mathrm{~s}$ prior to providing Aln-NEF to the subjects. The subjects avoided lying down until after a standardized meal was served 30 min following dosing. Each study dose was administered with the subject in a seated position. Dosing occurred between 8:00 AM and 9:00 AM. The study design is shown in Fig. 1.

\section{Safety and tolerability assessment}

Medical history and complete physical examinations were conducted during screening; an abbreviated physical examination was performed at discharge. Blood and urine samples were collected for routine laboratory tests at screening, during study periods, and at discharge. ECGs were obtained during screening and at discharge. Vital signs were monitored at screening and each day when the subjects were in-house. Subjects were continually observed and questioned during study periods for possible adverse events.

\section{Blood sampling times and bioanalytical methods}

Venous blood samples for determination of Aln plasma concentrations were collected prior to $(0 \mathrm{~h})$ and at $0.25,0.5,0.75$, $1,1.5,2,3,4,5,6,7$, and $8 \mathrm{~h}$ post-dose. The sample collection times for Aln analysis were based on a study by Yun, et al., who measured alendronate in plasma following administration of alendronate sodium tablets [9]. For determination of LT4 serum concentrations, venous blood samples were collected at baseline $(0.5,0.25$, and $0 \mathrm{~h}$ prior to dose $)$ and at 0.5 , $1,1.5,2,2.5,3,4,6,8,10,12,18,24$, and $48 \mathrm{~h}$ post-dose. Sample collection times for LT4 were those specified in the FDA guidance on LT4 tablets in vivo pharmacokinetic and bioavailability studies [8]. When both study drugs were administered concurrently, the sample collection and analysis procedures for each study drug were followed. Plasma and serum samples were frozen at $-70{ }^{\circ} \mathrm{C}$ and shipped on dry ice to the analytical facility.

Aln plasma concentrations were determined using a validated liquid chromatography method with a tandem mass spectrometric detection assay (LC-MS/MS). The assay had a lower limit of quantification (LLOQ) of $50.0 \mathrm{pg} / \mathrm{mL}$ and a linear range of 50.0-20,000 pg/mL. Precision and accuracy were 0.5 to $8.4 \%$ and -3.3 to $8.4 \%$, respectively. The selectivity of the assay was demonstrated during method validation. No significant interference at the retention time and mass transition of Aln or $\mathrm{d}_{6}$-Aln (internal standard) was observed from endogenous components in any of the 6 human plasma (EDTA) lots screened. The analytical method was developed by Celerion, Lincoln, Nebraska, and validated according to the standard operating procedures (SOPs) in effect at the laboratory. These SOPs were based on the good laboratory practice principles described in 21 CFR Part 58 and the Guidance 
Fig. 1 Study design

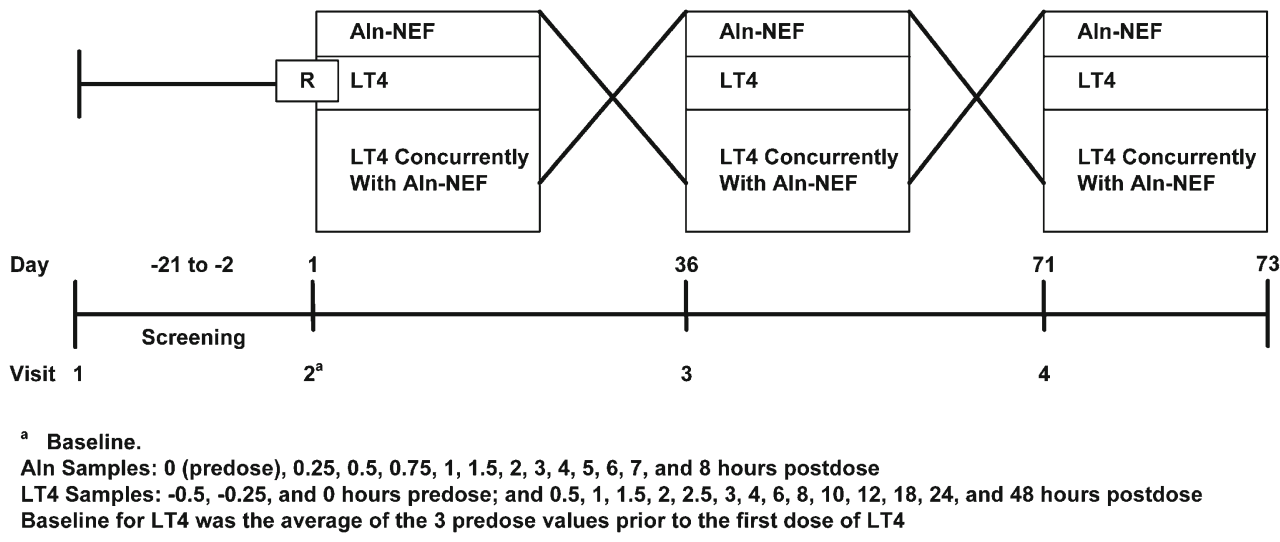

for Industry-Bioanalytical Method Validation (CDER, May 2001).

Total (bound and free) LT4 and T3 serum concentrations were determined using a Roche Diagnostics COBAS 6000, an approved analyzer for diagnostic use in the USA. The method is a validated electrochemiluminescence immunoassay.

The assay had an LLOQ of $1.91 \mu \mathrm{g} / \mathrm{dL}$ for LT4 and $48.05 \mathrm{ng} / \mathrm{dL}$ for T3. Linear ranges were 1.91-20.29 $\mu \mathrm{g} / \mathrm{dL}$ and 48.05-429.11 ng/dL for LT4 and T3, respectively. Precision and accuracy were 4.04 to $5.03 \%$ and -7.98 to $-1.84 \%$, respectively, for LT4 assay, and were 4.51 to $6.86 \%$ and -4.06 to $-2.51 \%$, respectively, for T3 assay.

All samples were analyzed at Celerion, Lincoln, NE

\section{Pharmacokinetic data analysis}

The primary pharmacokinetic parameters were $\mathrm{AUC}_{0-t}$ [area under the concentration-time curve from time zero to last measureable time point $(t)]$ and $\mathrm{C}_{\max }$ (maximum concentration). Individual plasma concentrations of Aln and serum concentrations of LT4 and T3 were used to estimate pharmacokinetic parameters using non-compartmental analysis methods. The $C_{\max }$ and time to reach the maximum concentration $\left(T_{\max }\right)$ were the observed values. $\mathrm{AUC}_{0-t}$ was calculated using the linear trapezoidal method and extrapolated to infinity for calculation of $\mathrm{AUC}_{0-\infty}$ as follows:

$\mathrm{AUC}_{0-\infty}=\mathrm{AUC}_{0-t}+\frac{\mathrm{AUC}_{\mathrm{est}}}{k_{\mathrm{el}}}$

where $C_{\text {est }}$ was the estimated concentration at the time of final quantifiable sample and $k_{\mathrm{el}}$ was the estimated rate constant at terminal phase and was calculated as the negative of the slope of the log:linear terminal portion of the concentration-time curve using linear regression. The terminal half-life $\left(T_{1 / 2}\right)$ was calculated as $0.693 / k_{\mathrm{el}}$.

Pharmacokinetic parameters of LT4 were calculated based on both baseline-corrected and uncorrected serum concentrations [10]. The average of the 3 serum concentrations at 0.5 , 0.25 , and $0 \mathrm{~h}$ prior to dosing was used as the baseline value and was subtracted from the LT4 serum concentration at each time point. Negative values after the correction were set to zero.

Pharmacokinetic analysis was conducted using a validated SAS® program.

\section{Statistical analysis}

The statistical analysis was carried out according to FDA guidelines on the intent-to-treat dataset that included all subjects for safety analysis and pharmacokinetic analysis. Summary statistics including mean, standard deviation, median, minimum, and maximum were provided for the demographic data and pharmacokinetic parameters. An analysis of variance (ANOVA) model, which included effects accounting for sequence, period, treatment, and subject nested in sequence, was performed on the log-transformed pharmacokinetic parameters $\left(C_{\max }\right.$ and AUC) to determine the relative bioavailability of a single dose of Aln-NEF with and without concurrent LT4, and the relative bioavailability of a single dose of LT4 with and without concurrent Aln-NEF. The geometric mean ratio, the difference between the expected log means, was estimated. Two-sided $90 \%$ confidence intervals (CIs) for the geometric mean ratio were computed. The study sample size was not based on a power calculation, but was considered to be adequate to characterize a potential interaction with sufficient accuracy based on previous similar studies and FDA guidance on LT4 tablets in vivo pharmacokinetic and bioavailability studies [8].

\section{Results}

\section{Demographics and disposition of subjects}

A total of 101 subjects were screened; 15 healthy men and 15 healthy women were enrolled. One subject withdrew after taking coadministered Aln-NEF and LT4; another subject was discontinued by the investigator for reasons other than 
safety after taking Aln-NEF alone and LT4 alone. Therefore, 28 subjects completed all 3 treatment periods of the study with no protocol deviations; 29 subjects received each of the 3 treatments. The mean $( \pm \mathrm{SD})$ age was $29.6( \pm 5.8)$ years, and mean $( \pm \mathrm{SD})$ body mass index $(\mathrm{BMI})$ was $24.53 \pm 2.48$. There were 15 Caucasians, 14 African Americans, and 1 Pacific Islander.

\section{Safety}

On study drug and for $48 \mathrm{~h}$ after study drug, the same low proportion of subjects had adverse events following administration of LT4 alone and Aln-NEF with LT4. No subjects reported adverse events following Aln-NEF alone. There were no serious adverse events, and no clinically important changes or trends in vital signs, ECGs, or clinical laboratory tests with any of the treatments. Most adverse events reported were mild to moderate and not related to treatment.

\section{Pharmacokinetic results}

Because the results were the same for the intent-to-treat and the per protocol analyses, only the intent-to-treat results are discussed. Aln plasma concentrations versus time profiles illustrate a rapid absorption phase after oral administration, followed by a multi-exponential disposition, and were superimposable between the treatments (Fig. 2).

The extent of absorption of Aln, determined by the $\mathrm{AUC}_{0-8}$, is similar between the treatments $(18,097 \mathrm{pg} \cdot \mathrm{h} / \mathrm{mL}$ for Aln-NEF alone, $17,784 \mathrm{pg} \cdot \mathrm{h} / \mathrm{mL}$ for Aln-NEF and LT4). Aln is eliminated rapidly from plasma with a mean estimated $T_{1 / 2}$ of $2.45 \mathrm{~h}$ after administration of Aln-NEF alone and $2.36 \mathrm{~h}$ after Aln-NEF and LT4 coadministration. This suggests a similar rate and extent of Aln absorption following oral administration of Aln-NEF alone and Aln-NEF with LT4 concurrently. Median $T_{\max }$ values were $0.75 \mathrm{~h}$ after both treatments; mean $( \pm \mathrm{SD}) C_{\max }$ were $11.6 \pm 6.07$ vs. $11.9 \pm 5.55 \mathrm{ng} / \mathrm{mL}$ and mean $( \pm \mathrm{SD}) \mathrm{AUC}_{0-\infty}$ were $18.6 \pm 8.95$ vs. $18.9 \pm 7.64 \mathrm{ng} \cdot \mathrm{h} / \mathrm{mL}$ after concurrent compared to single drug administration (Table 1).
The ANOVA for the ratio of $\mathrm{Aln} C_{\max }$ and $\mathrm{AUC}_{0-8}$ values after concurrent administration compared to single administration, using log-transformed data, are shown in Table 2 and illustrated in Fig. 3. The geometric mean ratio [(Aln-NEF + LT4)/Aln-NEF] was 0.91 (90\% CI 0.77-1.08) for $C_{\max }$ and 0.93 (90\% CI 0.79-1.08) for $\mathrm{AUC}_{0-8}$. Although the lower limit of the CIs for the mean ratios fell just below the usual bioequivalence acceptance range of $0.80-1.25$, the slight reduction in Aln exposure observed (approximately 9\%) when coadministered with LT4 was not considered clinically important.

These results suggest that concurrent administration of Aln with LT4 does not materially affect the pharmacokinetics of Aln.

The serum LT4 mean concentration-time profiles are shown in Fig. 3. There were no significant differences in serum LT4 concentrations at each observed time point between coadministration of Aln with LT4 and LT4 alone. Mean pharmacokinetic parameters were similar between treatments (Table 1).

The geometric mean ratios [(LT4 + Aln-NEF)/LT4] were 1.08 (90\% CI 1.01-1.15) for $C_{\max }$ and 1.05 (90\% CI 0.98 1.12) for $\mathrm{AUC}_{0-48}$ (Table 3). The CIs fell within the $0.8-1.25$ bioequivalence range.

LT4 serum concentration rises rapidly from baseline, followed by a slow decline. The mean $C_{\max }$ values are similar between LT4 alone $(3.50 \mu \mathrm{g} / \mathrm{dL})$ and Aln-NEF and LT4 $(3.76 \mu \mathrm{g} / \mathrm{dL})$. The extent of absorption, determined by $\mathrm{AUC}_{0-48}$, is similar between treatments $(95.40 \mu \mathrm{g} \cdot \mathrm{h} / \mathrm{dL}$ for LT4 alone, $101.0 \mu \mathrm{g} \mathrm{h} / \mathrm{dL}$ for Aln-NEF and LT4).

These results demonstrate that LT4 is bioequivalent when coadministered with Aln compared to LT4 administered alone; Aln does not affect the pharmacokinetics of LT4.

\section{Discussion}

Healthy male and female subjects were chosen as participants for this study in compliance with FDA guidance for in vivo
Fig. 2 Aln plasma concentrationtime profiles (mean $\pm \mathrm{SE}$, linear scale) Aln-NEF alone and AlnNEF and LT4 concurrently $(n=29)$

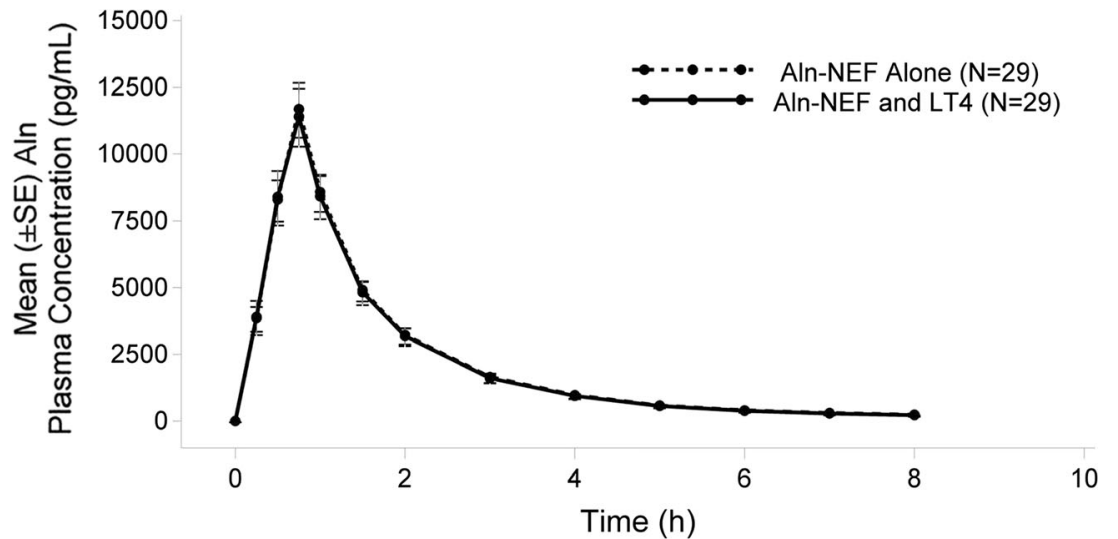


Table 1 Aln and LT4 mean $( \pm \mathrm{SD})$ pharmacokinetic parameters after oral administration of a single dose of $70 \mathrm{mg}$ Aln-NEF alone, $600 \mu \mathrm{g}$ LT4 alone, or $70 \mathrm{mg}$ Aln-NEF and $600 \mu \mathrm{g}$ LT4 concurrently in healthy subjects

\begin{tabular}{|c|c|c|c|}
\hline Parameter & Aln-NEF + LT4 $(n=29)$ & Aln-NEF $(n=29)$ & LT4 $(n=29)$ \\
\hline \multicolumn{4}{|c|}{ Aln } \\
\hline$C_{\max }(\mathrm{ng} / \mathrm{mL})$ & $11.6( \pm 6.07)$ & $11.9( \pm 5.55)$ & - \\
\hline$T_{\max }^{\mathrm{a}}(\mathrm{h})$ & $0.75(0.5-1.0)$ & $0.75(0.5-1.0)$ & - \\
\hline $\mathrm{AUC}_{0-8}(\mathrm{ng} \cdot \mathrm{h} / \mathrm{mL})$ & $17.8( \pm 8.62)$ & $18.1( \pm 7.43)$ & - \\
\hline $\mathrm{AUC}_{0-\infty}(\mathrm{ng} \cdot \mathrm{h} / \mathrm{mL})$ & $18.6( \pm 8.95)$ & $18.9( \pm 7.64)$ & - \\
\hline$T_{1 / 2}(\mathrm{~h})$ & $2.36( \pm 0.51)$ & $2.45( \pm 0.59)$ & - \\
\hline \multicolumn{4}{|c|}{ LT4 } \\
\hline$C_{\max }(\mu \mathrm{g} / \mathrm{dL})$ & $3.76( \pm 0.79)$ & - & $3.50( \pm 0.76)$ \\
\hline$T_{\max }^{\mathrm{a}}(\mathrm{h})$ & $3.0(1.0-10.0)$ & - & $2.5(1.0-12.0)$ \\
\hline $\mathrm{AUC}_{0-48}(\mu \mathrm{g} \cdot \mathrm{h} / \mathrm{dL})$ & $101.0( \pm 21.8)$ & - & $95.4( \pm 24.7)$ \\
\hline
\end{tabular}

${ }^{\mathrm{a}}$ Median and range pharmacokinetic and bioavailability studies using LT4 tablets. Most patients administered alendronate are postmenopausal women who are older than the subjects in this study. Alendronate is also approved in the USA for treatment to increase bone mass in men, so it was appropriate to include men in this study. The reason for any potential interaction between Aln and LT4 would be a physicochemical interaction of the products within the intestinal lumen. The physicochemical properties of the drugs are not a function of age and sex of the patients.

Aln dosing instructions specify upright posture, and LT4 dosing instructions do not specify posture. Therefore, all subjects were dosed in conformance with the Aln directions. Subjects were treated consistently at each dosing/draw visit. While there is some evidence that sustained standing versus supine posture can affect LT4 levels [11] and there are no such studies for Aln, the consistent seated position for this study provides a systematic control for any such variation, while remaining consistent with approved product labeling.

Bioanalytical methods used in the measurement of Aln have been reported previously $[12,13]$. Because absorbed Aln disappears rapidly from systemic circulation (plasma $T_{1 / 2}$ 0.5 to $2 \mathrm{~h}$ after oral administration) and is renally excreted rather than metabolized [14], the number of bioanalytical methods for use with serum or plasma is limited [15].
Consequently, many pharmacokinetic studies have relied on urine, rather than plasma, in the determination of Aln concentrations $[16,17]$. To date, there are 2 studies that have determined plasma Aln concentrations for bioavailability and bioequivalence assessments of Aln tablets using a validated highperformance liquid chromatography (HPLC) method with fluorescence detection and LLOQs of 1 to $2 \mathrm{ng} / \mathrm{mL}[9,18]$. In the present study, a validated LC-MS/MS method with high sensitivity (LLOQ $50 \mathrm{pg} / \mathrm{mL}$ ) and selectivity was used to determine Aln plasma concentrations.

In the 2 previously published studies $[9,18]$, Aln tablets were administered with $240 \mathrm{~mL}$ of water after an overnight fast; a standardized meal was given at $4 \mathrm{~h}$ post-dose. In the present study, Aln-NEF was dissolved in $4 \mathrm{oz}$ (about $120 \mathrm{~mL}$ ) water for administration; a standardized meal was given 30 min after dosing. The $T_{\max }$ and $T_{1 / 2}$ values obtained in this study were similar to those previously reported $[9,18]$. Aln plasma exposure values $\left(C_{\max }\right.$ and $\left.\mathrm{AUC}_{0-\infty}\right)$ were slightly lower, which may reflect differences in population studied, bioanalytical method used, drug administration, or timing of the first meal.

In the present study, there was no material pharmacokinetic interaction between Aln and LT4 observed when Aln-NEF and LT4 were coadministered. The $90 \%$ CIs for the geometric mean ratio of $\mathrm{Aln} C_{\max }$ (0.77 to 1.08$)$ and $\mathrm{AUC}_{0-\infty}$ (0.79 to

Table 2 Relative geometric mean and ratio of Aln-NEF and LT4 concurrently to Aln-NEF alone for alendronate

\begin{tabular}{|c|c|c|c|c|c|}
\hline \multirow[t]{2}{*}{ Pharmacokinetic parameter (units) } & \multicolumn{2}{|l|}{ Estimated geometric mean ${ }^{\mathrm{a}}$} & \multirow[t]{2}{*}{ Ratio of means ${ }^{\mathrm{b}}$} & \multicolumn{2}{|l|}{$90 \% \mathrm{CI}$} \\
\hline & Aln-NEF + LT4 $(n=29)$ & Aln-NEF $(n=29)$ & & Lower limit & Upper limit \\
\hline$C_{\max }(\mathrm{pg} / \mathrm{mL})$ & 9964 & 10,922 & 0.912 & 0.773 & 1.077 \\
\hline $\mathrm{AUC}_{0-8}(\mathrm{pg} \cdot \mathrm{h} / \mathrm{mL})$ & 15,576 & 16,798 & 0.927 & 0.795 & 1.081 \\
\hline
\end{tabular}

${ }^{a}$ Estimated geometric means are from the statistical model using terms for sequence, subject, period, and treatment

b $\mathrm{Aln}-\mathrm{NEF}+\mathrm{LT} 4$

Aln-NEF 
Fig. 3 Total LT4 mean serum concentration-time profiles (baseline-corrected, mean $\pm \mathrm{SE}$, linear scale) LT4 alone and AlnNEF and LT4 concurrently $(n=29)$

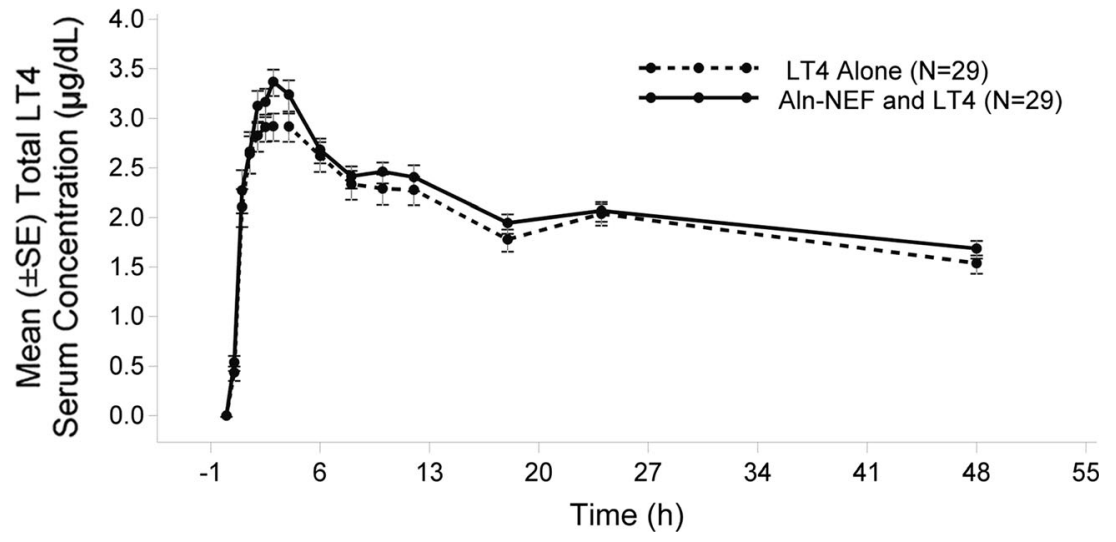

1.08) contained 1 , the lower bounds were near the bioequivalence boundary of 0.8 , and the upper bounds were within the boundary of 1.25 . The $90 \%$ CIs for the geometric mean ratio of LT4 $C_{\max }$ (1.01 to 1.15$)$ and $\mathrm{AUC}_{0-48}(0.98$ to 1.12$)$ fell within the 0.8 to 1.25 bioequivalence boundary. The variability in $T_{\max }$ was greater than anticipated. The reason for this higher variability is unknown. Nevertheless, AUC values were much tighter and are far more important. These results are consistent with the prediction based on the known physicochemical properties of Aln-NEF and LT4, and the pharmacokinetic characteristics of Aln and LT4.

Because LT4 is administered daily and Aln-NEF is administered once weekly, an increase of a few percent in LT4 absorption 1 day of the week would have no appreciable effect on average LT4 levels. These results indicate that coadministration of LT4 with the specific Aln-NEF formulation tested is feasible. The LT4 levels thereby achieved were bioequivalent to those achieved in the same study subjects in the absence of Aln. Of course, monitoring of thyroid function tests and adjustment of LT4 dosage, as needed, will remain essential in the management of hypothyroidism. It would be appropriate to perform laboratory reassessment of the patient's thyroxine status after 6 to 8 weeks of coadministration. The Aln levels achieved in this study missed the formal boundary for determination of bioequivalence by less than $1 \%$. In the clinical trial (FIT) that established the anti-fracture efficacy of Aln, the participants in the active treatment arm received $5 \mathrm{mg}$ daily for the first 2 years, followed by $10 \mathrm{mg}$ daily for approximately one more year [1]. In the extension of that study, (FLEX) participants received either 5 or $10 \mathrm{mg}$ daily or placebo. The 2 active arms produced similar results and were pooled [19]. It seems clear that there is a much wider therapeutic window for Aln treatment of osteoporosis than for LT4 treatment of hypothyroidism. Therefore, no clinically important difference in the efficacy of Aln would be expected to result from coadministration.

For LT4, $C_{\max }$ is marginally higher for the combined administration group (CI for the ratio was 1.006-1.148). Of course, in view of the long half-life of LT4, it is the AUC that is of clinical importance. The upper limit of the confidence interval for the ratio of AUCs was 1.119, and the mean ratio was 1.049. Taking into account the weekly schedule of administration of Aln, and the usual daily administration schedule for LT4, the effect on weekly AUC of LT4 would be less than $1 \%$ at the mean and less than $2 \%$ at the upper limit of the $90 \%$ confidence interval.

Aln-NEF and LT4 were well tolerated when given alone or concurrently administered in healthy subjects. There was no significant pharmacokinetic interference between the AlnNEF formulation of Aln sodium and LT4. LT4 does not materially affect absorption of Aln when administered as AlnNEF. Whether the findings of this study that used Aln-NEF apply to any other formulations of Aln is uncertain. A formal interaction study would be necessary to evaluate each

Table 3 Geometric mean and ratio of Aln-NEF and LT4 concurrently to LT4 alone for LT4

\begin{tabular}{llllll}
\hline Pharmacokinetic parameter (units) & \multicolumn{2}{l}{ Estimated geometric mean ${ }^{\mathrm{a}}$} & & \multirow{2}{*}{ Ratio of means $^{\mathrm{b}}$} & \multicolumn{2}{c}{$90 \%$ CI } \\
\cline { 2 - 5 } & Aln-NEF + LT4 $(n=29)$ & Aln-NEF $(n=29)$ & & Lower limit & Upper limit \\
\hline$C_{\max }(\mu \mathrm{g} / \mathrm{dL})$ & 3.694 & 3.437 & 1.075 & 1.006 & 1.148 \\
$\mathrm{AUC}_{0-48}(\mu \mathrm{g} \cdot \mathrm{h} / \mathrm{dL})$ & 97.457 & 92.931 & 1.049 & 0.983 & 1.119 \\
\hline
\end{tabular}

${ }^{a}$ Estimated geometric means are from the statistical model using terms for sequence, subject, period, and treatment

$\mathrm{b} \underline{\mathrm{Aln}-\mathrm{NEF}+\mathrm{LT} 4}$ 
formulation of Aln, available as either tablets for oral use or oral solution, with LT4 as the formulations may vary in composition.

Acknowledgements This study was sponsored and funded by Mission Pharmacal Company, San Antonio, TX.

Compliance with ethical standards This study was conducted in compliance with the World Medical Association Declaration of HelsinkiEthical Principles for Medical Research Involving Human Subjects and Good Clinical Practice. The protocol was reviewed by an institutional review board. Written informed consent was obtained from all subjects before enrolment in the study.

Informed consent Informed consent was obtained from all individual participants included in the study.

Conflicts of interest HGB has acted as a consultant to Merck, Amgen, Radius Health and Mission Pharmacal Company, and as an investigator for Merck \& Co and Amgen. MAW was previously employed by Mission Pharmacal Company. MEH has acted as a consultant to Mission Pharmacal Company. SE had served as a consultant to Mission Pharmacal Company and as a consultant and on the advisory panel for Merck Pharmaceuticals.

Open Access This article is distributed under the terms of the Creative Commons Attribution-NonCommercial 4.0 International License (http:// creativecommons.org/licenses/by-nc/4.0/), which permits any noncommercial use, distribution, and reproduction in any medium, provided you give appropriate credit to the original author(s) and the source, provide a link to the Creative Commons license, and indicate if changes were made.

\section{References}

1. Black DM, Cummings SR, Karpf DB, Cauley JA, Thompson DE, Nevitt MC, Bauer DC, Genant HK, Haskell WL, Marcus R, Ott SM, Torner JC, Quandt SA, Reiss TF, Ensrud KE (1996) Randomised trial of effect of alendronate on risk of fracture in women with existing vertebral fractures. Fracture Intervention Trial Research Group Lancet 348:1535-1541

2. Fosamax Prescribing Information, Merck, revised February 2012.

3. Binosto Prescribing Information, Mission Pharmacal Company, revised August 2013.

4. PDR3D Digital Drug Database. Retrieved from http://www.pdr3d. $\mathrm{com} /$

5. Qato DM, Alexander GC, Conti RM, Johnson M, Schumm P, Lindau ST (2008) Use of prescription and over-the-counter medications and dietary supplements among older adults in the United States. JAMA 300(24):2867-2878

6. Synthroid PI, AbbVie Inc., September 2012.

7. U.S. Food and Drug Administration, Center for Drug Evaluation and Research, Office of Clinical Pharmacology and Biopharmaceutics. Approval Package for Application Number NDA 21-402: Synthroid Clinical Pharmacology and Biopharmaceutics Review, Executive Summary. Accessed January 11, 2017 from http://www.accessdata.fda. gov/scripts/cder/daf.

8. Guidance for Industry: Levothyroxine sodium tablets - in vivo pharmacokinetic and bioavailability studies and in vitro dissolution testing. FDA, February 2001.

9. Yun MH, Woo JS, Kwon KI (2006) Bioequivalence and pharmacokinetics of $70 \mathrm{mg}$ alendronate sodium tablets by measuring alendronate in plasma. Arch Pharm Res 29(4):328-332

10. Bolton S (2005) Bioequivalence studies for levothyroxine. AAPS J 7(1):E47-E53

11. Abalan F, Hui Bon Hoa A, Dufrechou N, Ellison W, Rigal F, Perey F, Combourieu I, Bourgeois M (1991 Aug) Effect of posture on total thyroxine plasma concentration. Horm Metab Res 23(8): 404-405

12. Lin JH (1996) Bisphosphonates: a review of their pharmacokinetic properties. Bone 18:75-85

13. Sparidans RW, den Hartigh J (1999) Chromatographic analysis of bisphosphonates. Pharm World Sci 21(1):1-10

14. Porras AG, Holland SD, Gertz BJ (1999) Pharmacokinetics of alendronate. Clin Pharmacokinet 36:315-328

15. Zhu LS, Lapko VN, Lee JW, Basir YJ, Kafonek C, Olsen R, Briscoe C (2006) A general approach for the quantitative analysis of bisphosphonates in human serum and urine by high-performance liquid chromatography/tandem mass spectrometry. Rapid Commun Mass Spectrom 20:3421-3426

16. Tarcomnicu I, Silvestro L, Savu SR, Gherase A, Dulea C (2007) Development and application of a high-performance liquid chromatography-mass spectrometry method to determine alendronate in human urine. J Chromatogr A 1160:21-33

17. Apostolou C, Dotsikas Y, Kosoulos C, Tsatsou G, Colocouri F, Soumelas GS, Loukas YL (2007) Application of a semiautomated 96-well format solid-phase extraction, columnswitching, fluorescence detection protocol for the determination of alendronate in human urine samples obtained from a bioequivalence study. J Pharm Biomed Anal 43(3):1151-1155

18. Rhim SY, Park JH, Park YS, Lee MH, Kim DS, Shaw LM, Yang SC, Kang JS (2009) Bioavailability and bioequivalence of two oral formulations of alendronate sodium $70 \mathrm{mg}$ : an open-label, randomized, two-period crossover comparison in healthy Korean adult male volunteers. Clin Therapeutics 31(5):1037-1045

19. Black DM, Schwartz AV, Ensrud KE, Cauley JA, Levis S, Quandt SA, Satterfield S, Wallace RB, Bauer DC, Palermo L, Wehren LE, Lombardi A, Santora AC, Cummings SR, FLEX Research Group (2006) Effects of continuing or stopping alendronate after 5 years of treatment: the Fracture Intervention Trial Long-term Extension (FLEX): a randomized trial. JAMA 296(24):2927-2938 